\title{
Maximum MBF Types
}

\author{
Tkachenco V. G. ${ }^{1, *}$, Sinyavsky $O$. V. $^{2}$ \\ ${ }^{1}$ Institute of Radio Television Electronics, Odessa National Academy of Telecommunications, Ukraine \\ ${ }^{2}$ Department of Fundamental Sciences, Odessa Military Academy, Ukraine
}

Copyright $(2018$ by authors, all rights reserved. Authors agree that this article remains permanently open access under the terms of the Creative Commons Attribution License 4.0 International License

\begin{abstract}
The classification of monotone Boolean functions (MBF) into types is given. The notion of the maximum type of MBF is introduced, and the shift-sum types are constructed. The matrices of type distribution by rank are constructed. Convenient algorithms for finding the number of maximum types and the maximum types themselves are presented. The proposed methods can be used to analyze large MBF ranks.
\end{abstract}

Keywords Monotone Boolean Functions, Disjunctive Complement, Conjunctive Complement, MBF Types, Maximum Types, MBF Profile, Free Distributive Lattice

\section{Introduction}

In 1997 Engel entered the concept of the profile of monotone Boolean function [1]. He gave this definition:

Definition. Given an n-variable MBF f where $f \neq 1$, the profile of $\mathrm{f}$ is a vector of length $\mathrm{n}\left(a_{1}, a_{2}, \ldots, a_{n}\right)$, where the ith entry is equal to the number of minimal terms of $f$ which are i-sets.

To find the number of nonisomorphic MBFs, T.Stephen and T.Yusun [2] partitioned into classes the all set of MBFs using these profiles. They developed an algorithm on Matlab to calculate the number of classes of such MBFs of 7 variables. This method is based on the partitioning of MBF on profiles, which are defined in [1]. Apart from using for MBFs, profiles are used for research with simplicial complexes in topology.

Independently, we the concept of MBF type was introduced [3]. In [4] a classification of MBF types was developed. The application of this method gave new results. Using MBF types, a cryptosystem based on triangular codes was constructed in [5]. In [6] a recursive construction of n-gonal codes was obtained. In [7] a method for constructing MBF blocks developed. In [8], a new method of recursive calculation of the Dedekind number [9] was developed on the basis of MBF blocks and the algebraic properties of MBF blocks were researched.
In this paper we introduce the concept of the maximum type, which was not previously encountered in the literature. Maximum types are easier to compute than all types of $\mathrm{MBF}$ and, as a consequence, the number of all MBF s of a definite rank, i.e. computation of the Dedekind number.

In this paper we give two algorithms. 1 - computation the number of maximum types of MBF and 2 - enumeration of all maximum types of a certain rank.

\section{Results}

A Boolean function of $\mathrm{n}$ elements is a mapping $f: B^{n} \rightarrow B$, where $B=\{0,1\}$. A monotonic Boolean function (MBF) is a Boolean function under the condition: for any $\alpha, \beta \in B^{n}$ such that $\alpha<\beta, f(\alpha)<f(\beta)$. The lattice of all MBFs of rank $n$ is a free distributive lattice of rank $n$ and add zero MBF (on all sets is equal to 0 ) and a unit MBF (on all sets is equal to 1 ) MBF. Under the ratio of greater then or less then we mean the order in this lattice of all MBF. The Boolean function we will be considered in disjunctive normal form (DNF). It is possible to define MBF through DNF. A monotone Boolean function is a Boolean function that does not have a negation operation in the form of DNF, but only operations of disjunction and conjunction.

Define a minimal term of an MBF $f$ to be an input $\mathrm{x} \in\{0$, 1 ) $\mathrm{n}$ such that $f(x)=1$ and $f(y)=0$ if $\mathrm{y}<\mathrm{x}$. The minimal terms of a monotone Boolean function represent the "smallest" sets where the function equals one - any input below $\mathrm{x}$ evaluates to zero, and everything above evaluates to one by virtue of monotonicity. For example, the function $f\left(x_{1} x_{2} x_{3}\right)=x_{1} x_{2} x_{3}$ evaluates to one at $(1,0,0)$ and $(0,1,1)$, as well as at vectors above $(1,0,0)$ and $(0,1,1)$, and evaluates to 0 at all other vectors. Indeed, each MBF can be written as a disjunction of clauses, each representing one of its minimal terms.

A vector $T=\left(a_{0}, a_{1}, \ldots, a_{i}, \ldots, a_{n}\right)$ is an MBF type if the $\mathrm{i}$-th component of the vector ai is equal to the number of 
conjunctive clauses in the form of DNF, which consist of $i$ variables, i.e. have length i. Several new characteristics were introduced for the type: the number $\mathrm{n}$ is called the rank of a type $\mathrm{T}$; the number of non-zero component $\mathrm{v}-$ weight of a type $T$; the number $j$ of the first nonzero components on the right - the right border of the $\mathrm{T}$; the number $\mathrm{j}$ of the first nonzero components on the left - the left border of the $\mathrm{T}$; the sum $\mathrm{m}$ of all the components of the $\mathrm{T}$ - cardinality type $\mathrm{T}$.

The MBF type is defined as the profile, except that the MBF type has an additional component $a_{0}$, with which it is possible to describe all MBFs of this rank, in particular, a unit MBF. This component is equal to one only for the unit function, and the remaining components in this case are equal to zero. Ie the unit function is represented by one conjunctive clauses of zero length, while the zero function has 0 clauses.

And with this additional component, the MBF type becomes symmetrical, which allows us to introduce a new operation, which we call a shift-sum. Using this operation, types can be calculated recurrently, using types of the previous rank. Ie types of rank $n$ are recursively computed through types of rank $n-1$. Thus algorithms for enumeration of types of a certain rank are simplified. Because of asymmetry of the profile with it it is more difficult to perform such operation.

The type $T$ is called maximal if, with increasing any of its components by 1 , the resulting vector will not be a type, i.e. there is no MBF, the type of which would be equal to this vector. Conversely, if we subtract one or more units from any component, the resulting vector will also be an MBF type, since we will remove the clauses of the length of the corresponding component from the existing MBF. By definition, this function will be monotonic. Thus, any type can be obtained by subtracting integers from components of one or more maximum types.

We call type $T_{1}^{-1}=\left(a_{n}, a_{n-1}, \ldots, a_{n-i}, \ldots, a_{1}, a_{0}\right)$ the inverse type for type $T_{1}=\left(a_{0}, a_{1}, \ldots, a_{i}, \ldots, a_{n}\right)$. This means that if the MBF $f$ is of type $T_{1}$, then the type of disjunctive complement MBF $g$ is $T_{1}^{-1}$

EXAMPLE 1. We take the MBF $\mathrm{f}$ from 5 variables equal to one on the input sets 00011, 00111, 01011, 10011, 01111, 10111, 11011, 11100, 11101, 11110 and 11111 . The minimal term of the function $\mathrm{f}$ are the sets 00011 and 11100 , the first of which is at level 3 , and the second at level 2 of the Boolean cube. In the symbolic form, the MBF looks like $f=x_{2} x_{1} \vee x_{5} x_{4} x_{3}$. Hence the type $\mathrm{T}(\mathrm{f})$ of $\mathrm{f}$ is $(0,0,1,1,0,0)$. The rank of this type is $\mathrm{n}(\mathrm{T})=5$, the weight is $v(T)=2$, the left boundary is $i(T)=2$, the right boundary is $\mathrm{j}(\mathrm{T})=3$. The same type has a number of MBFs, in particular MBF with minimal term 00101 and 11001.

EXAMPLE 2. The following are the maximum types of ranks from 0 to 4 .

For rank 0 , only type (1) is maximal.

For rank 1, the maximal are 2 types: $(0,1)$ and $(1,0)$.
For rank 2, the maximal is 3 types: $(0,0,1),(0,2,0)$ and $(1,0,0)$.

For rank 3 , the maximal is 5 types: $(0,0,0,1),(0,0,3,0)$, $(0,1,1,0),(0,3,0,0)$, and ( 1.0 .0 .0$)$.

For rank 4 , the maximal is 10 types: $(0,0,0,0,1),(0,0,0$, $4,0),(0,0,1,2,0),(0,0,3,1,0),(0,0,6,0,0),(0,1,0,1$, $0),(0,1,3,0,0),(0,2,1,0,0),(0,4,0,0,0),(1,0,0,0,0)$

$$
\begin{array}{ccc}
\text { We call the types of views } \\
(0,0, \ldots, 0),(1,0, \ldots, 0),(0,0, \ldots, 0,1) & \text { zero, left and }
\end{array}
$$

right, respectively. For the zero type, the right and left units coincide and are equal to (1).

Let's call a pair of types $T_{1}$ and $T_{2}$ rank $n$ admissible if the right boundary of $j\left(T_{1}\right)$ is strictly less than the left boundary of $i\left(T_{2}\right)$, i.e. All conjunctive clauses of MBF of type $T_{1}$ are strictly less than any clauses of MBF of type $T_{2}$.

For any admissible pair of types, a shift-sum operation is defined:

$$
\begin{aligned}
& T=T_{1} \circ T_{2}=\left(a_{0}, a_{1}, \ldots, a_{n}\right) \circ\left(b_{0}, b_{1}, \ldots, b_{n}\right)= \\
& =\left(b_{0}, a_{0}+b_{1}, \ldots, a_{n-1}+b_{n}, a_{n}\right)=\left(c_{0}, c_{1}, \ldots, c_{n+1}\right)
\end{aligned}
$$

If $T=T_{1} \circ T_{2}, T^{-1}=T_{2}^{-1} \circ T_{1}^{-1}$

Such an operation with a zero vector $T_{0}=(0,0, \ldots, 0)$ it is possible to carry out both on the right, and at the left:

$$
\begin{aligned}
& T=T_{1} \circ T_{0}=\left(a_{0}, a_{1}, \ldots, a_{n}\right) \circ(0,0, \ldots, 0)=\left(0, a_{0}, \ldots, a_{n-1}, a_{n}\right) \\
& T=T_{0} \circ T_{1}=(0,0, \ldots, 0) \circ\left(a_{0}, a_{1}, \ldots, a_{n}\right)=\left(a_{0}, \ldots, a_{n-1}, a_{n}, 0\right)
\end{aligned}
$$

LEMMA 1. The shift-sum operation over two admissible maximum types of rank $n-1$ gives a maximum type of rank $n$.

Proof. To do this, it suffices to show that a function that corresponds to a shift-sum of two types $T_{1}$ and $T_{2}$ $T=T_{1} \circ T_{2}$ is a function $f(n)=f_{1}(n-1) x_{n} \vee f_{2}(n-1)$ and this function is monotone, here $f(n)$ is of type $T$, $f_{1}(n-1)-T_{1}, f_{2}(n-1)-T_{2}$. Since $T_{1}$ and $T_{2}$ constitute an admissible pair, the length of any conjunctive clauses of $f_{1}(n-1)$ is strictly less than the length of any clauses of $f_{2}(n-1)$. After the operation $f_{1}(n-1) x_{n+1}$ the lengths will not be strictly less; the highest variable is added. Therefore, the type of MBF $f(n)$ is

$$
\begin{aligned}
T & =T_{1} \circ T_{2}=\left(a_{0}, \ldots, a_{i}, 0, \ldots, 0\right) \circ\left(0, \ldots, 0, b_{i+1}, \ldots, b_{n-1}\right)= \\
& =\left(0, a_{0} \ldots, a_{i}+b_{i+1}, \ldots, b_{n-1}, 0\right)
\end{aligned}
$$

Obviously, this type will be maximal. Indeed, we take some MBF $f(n)$ corresponding to type $T$. Clauses 
$f(n)$ that contain $x_{n}$ are of type $T_{1}$, the others are of type $T_{2}$. Whichever conjunctive clauses we add to $f(n)$, it will either contain $x_{n}$ or not. In the first case, it is of type $T_{1}$ and then this type is not maximal, and in the second case - to type $T_{2}$, then type $T_{2}$ is not maximal. But by the hypothesis of the lemma they are maximal. Therefore, the conjunctive clauses cannot be added to the MBF $f(n)$, and therefore the corresponding type $T$ is maximal.

Here it should be noted that the left and right units cannot be obtained by a shift-sum of two maximum types, but only with the help of such equalities:

$$
\begin{aligned}
& (0,0, \ldots, 0,0) \circ(1,0, \ldots, 0,0)=(1,0, \ldots, 0,0) \\
& (0,0, \ldots, 0,1) \circ(0,0, \ldots, 0,0)=(0,0, \ldots, 0,1)
\end{aligned}
$$

The lemma is proved.

Let's consider the type $T=\left(a_{0}, \ldots, a_{i}, \ldots, a_{n}\right)$ of rank $n$. We take a vector $T_{2}$ of rank n-1 with a left boundary $i$, i.e. $T_{2}=\left(0, \ldots, 0, c_{i}, c_{i+1}, \ldots, c_{n-1}\right)$. If all components from $i+1$ to $n-1$ of the vector $T_{2}$ coincide with the corresponding components of type $T$, and $i$ the component of the vector $T_{2}$ is greater than zero and less than or equal to the $i$-th component of the type, i.e. $0<c_{i} \leq a_{i}, \quad c_{k}=a_{k}, k=\overline{i+1, n-1}$, then we call such a vector $T_{2}$ a right part of type $T$.

Next, consider a vector $T_{1}$ of rank $n-1$ with a right boundary $j$, i.e. $T_{1}=\left(b_{0}, b_{1} \ldots, b_{j}, 0, \ldots, 0\right)$. If all components from zero to $j-1$ of vector $T_{1}$ coincide with the components from first to $j$ of type $T$, and $j$ component of the vector $T_{1}$ is greater than zero and is less than or equal to the $j+1$ component of the type $T$, i.e. $0<b_{j} \leq a_{j+1}, b_{k}=a_{k+1}, k=\overline{0, j-1}$, then we call such a vector a left part of type $T$.

If $p$ is a cardinality of type $T$, then the left (right) part can have cardinality from 0 to $p$. Hence, one number - the cardinality of the left (right) part, from 0 to $p$, completely determines the left (right) part. By definition, any left part is a type of rank $n$ when adding the zero component on the left, and the right part is a type of rank $n$ when adding the zero component to the right. Note that not every left (right) part can be a type of rank $n-1$. But if the left (right) part is a type, then all left (right) parts of smaller cardinality are type of rank $n-1$. Consequently, there is a maximal left (right) part, which is a type of rank $n-1$. In some cases, the cardinality of the maximfl left (right) part can coincide with the cardinality of type $T$. Thus, the maximal right (left) part of type $T$ is uniquely determined, as the right (left) part of type $T$ of maximum cardinality, which is also a type of rank $n-1$.

LEMMA 2. For any type $T$ of rank $n$ and cardinality $p$, for its maximal left side $T_{1}$ of cardinality $p_{1}$ and maximal right side $T_{2}$ of cardinality $p_{2}$, carry out $p_{1}+p_{2} \geq p$.

Proof. Let's choose $f(n)$ of type $T$ such that its $p_{1}$ conjunctive clause corresponding to the maximal left part $T_{1}$ contain the variable $x_{n}$ and all the other clauses do not contain $x_{n}$. Then the clauses from which the variable $x_{n}$ is removed form an MBF $f_{1}(n-1)$ of type $T_{1}$ Assume that $p_{1}+p_{2}<p$, then the MBF $f_{2}(n-1)$, corresponding to the maximal right part of $T_{2}$, consists of clauses that do not contain $x_{n}$ and in $f(n)$ is are clauses without $x_{n}$ that do not belong to either $f_{1}(n-1)$ or $f_{2}(n-1)$. Adding these clauses to $f_{2}(n-1)$, we obtain $f_{2}^{\prime}(n-1)$ of type $T_{2}^{\prime}$. Type $T_{2}^{\prime}$ is a right part of type $T$ and has a cardinality greater than type $T_{2}$, which contradicts the definition of the maximal right part $T_{2}$. Therefore, assumption $p_{1}+p_{2}<p$ is false and carried out $p_{1}+p_{2} \geq p$. The lemma is proved.

Complement $D_{1}$ of a right part $T_{2}$ of type $T$ is a vector T of rank $n-1$, obtained by subtracting components from 0 to $n-1$ of the vector $T_{2}$ from components from the first to the $\mathrm{n}$-th of type $\mathrm{T}$. In the construction of the complement $D_{1}$, we drop the zero component of the vector $\mathrm{T}$. If the zero component of the complement is zero, then, drop this zero component from the complement, we obtain a vector of n-1-th $\operatorname{rank} D_{1}$. The complement $D_{1}$ is a left part of type $\mathrm{T}$ and with vector $T_{2}$ constitute an admissible pair $D_{1} \circ T_{2}=T$.

Complement $D_{2}$ of the left part $T_{1}$ of type $\mathrm{T}$ is the vector of rank $n-1$, obtained by subtracting components from 0 to $n-1$ of the vector $T_{1}$ from components from 1 to $\mathrm{n}$ of type $\mathrm{T}$. In constructing the complement $D_{2}$, discarding this $\mathrm{n}$-th component from the complement, we obtain a vector of the n-1-th $\operatorname{rank} D_{2}$. The complement $D_{2}$ is a right part of type $\mathrm{T}$ and with vector $T_{1}$ constitute an admissible pair of rank $n-1$.

Let $p_{1}$ be the cardinality of the maximal left side $T_{1}$ and let $p_{2}$ be the cardinality of the maximal right part $T_{2}$. Let p3 be the cardinality of the complement of $D_{1}$ to $T_{2}$ and $\mathrm{p} 4$ the cardinality of the complement of $D_{2}$ to $T_{1}$. Then the cardinality equality $p_{3}+p_{2}=p_{1}+p_{4}=p$ holds. This follows from the definitions of the complements. Further, taking into account that $p_{1}+p_{2} \geq p$, we get that 
the complement is less than the right (left) maximum part and, consequently, is a type.

Thus, it follows from Lemma 2 and the definition of the complement that the complement $D_{1}\left(D_{2}\right)$ of the maximal right (left) part $T_{2}\left(T_{1}\right)$ of type T rank $\mathrm{n}$ is a type rank $n-1$. In addition, the decompositions of $D_{1} \circ T_{2}=T$ and $T_{1} \circ D_{2}=T$ are the only right and unique left decompositions of type $\mathrm{T}$ rank $\mathrm{n}$ into 2 admissible types rank $n-1$. It will be shown below that for the maximum types these two decompositions coincide.

LEMMA 3. Any maximum type of the $n$-th rank is uniquely represented as a shift-sum of two maximum types of n-1 rank.

Proof. From the maximum type of the $\mathrm{n}$-th rank $\mathrm{T}$ we select the maximal left part $T_{1}$ and the maximal right part $T_{2}$. Let $D_{1}$ be the complement for $T_{2}$, and $D_{2}-$ for $T_{1}$. Then, as shown above, $T_{1} \circ D_{2}=T$ and $D_{1} \circ T_{2}=T$. Let's prove that $D_{2}=T_{2}$ and $D_{1}=T_{1}$ respectively. Assume that $D_{2} \neq T_{2}$. Then two cases are possible: when $T_{2}<D_{2}$ and $T_{2}>D_{2}$.

The first case. Because $D_{2}$ is of type rank n-1, then it cannot be greater than the maximal right part $T_{2}$.

The second case. Let's take MBF $f_{1}(n-1)$ of type $T_{1}$ and MBF $f_{2}(n-1)$ of type $D_{2}$. Then all conjunctive clauses $f_{1}(n-1)$ by definition of the complement have length less than the clauses of $f_{2}(n-1)$. Let's take MBF $f_{2}^{\prime}(n-1)$ of type $T_{2}$ such that $f_{2}^{\prime}(n-1)>f_{2}(n-1)$. Since $T_{2}>D_{2}$, then $f_{2}^{\prime}(n-1)$ contains clauses that are absent in $f_{2}(n-1)$. But the shift-sum $T_{1} \circ D_{2}=T$ already gives the maximum type. Consequently, it is impossible to add an additional conjunction and the second case also leads to a contradiction.

Hence it follows that $D_{2}=T_{2}$. Consequently, types $T_{1}$ and $T_{2}$ constitute admissible pair $T=T_{1} \circ T_{2}$.

Next, we prove that these types are maximal. Let the type $T_{1}$ is not maximal. Then, by what has been proved before, it can be obtained from some maximum type by subtracting units from its components. Let's substitute this maximum type instead of type $T_{1}$ and we get some type $T^{\prime}$ that contains conjunctive clauses not belonging to type $\mathrm{T}$. But this can not be, because, by definition, type $\mathrm{T}$ is the maximal. The lemma is proved.

It follows from Lemmas 2 and 3 that for any type $\mathrm{T}$ of rank $\mathrm{n}$ and cardinality $\mathrm{p}$ having the maximal left side of cardinality $\mathrm{p} 1$ and the maximal right part of cardinality $\mathrm{p} 2$ there are $\mathrm{p} 1+\mathrm{p} 2-\mathrm{p}+1$ admissible decomposition of type $\mathrm{T}$ on types $\mathrm{T} 1$ and $\mathrm{T} 2$ of rank $\mathrm{n}-1$.

EXAMPLE 3. From maximum types MBF 4th rank, using the shift-sum, we obtain the maximum types of MBF of 5 th rank. Let us write them out in lexicographical order.

$$
\begin{aligned}
& (0,0,0,0,1) \circ(0,0,0,0,0)=(0,0,0,0,0,1) \\
& (0,0,0,4,0) \circ(0,0,0,0,1)=(0,0,0,0,5,0) \\
& (0,0,1,2,0) \circ(0,0,0,0,1)=(0,0,0,1,3,0) \\
& (0,0,3,1,0) \circ(0,0,0,0,1)=(0,0,0,3,2,0) \\
& (0,0,6,0,0) \circ(0,0,0,0,1)=(0,0,0,6,1,0) \\
& (0,0,6,0,0) \circ(0,0,0,4,0)=(0,0,0,10,0,0) \\
& (0,1,0,1,0) \circ(0,0,0,0,1)=(0,0,1,0,2,0) \\
& (0,1,3,0,0) \circ(0,0,0,0,1)=(0,0,1,3,1,0) \\
& (0,1,3,0,0) \circ(0,0,0,4,0)=(0,0,1,7,0,0) \\
& (0,2,1,0,0) \circ(0,0,0,0,1)=(0,0,2,1,1,0) \\
& (0,2,1,0,0) \circ(0,0,0,4,0)=(0,0,2,5,0,0) \\
& (0,4,0,0,0) \circ(0,0,0,0,1)=(0,0,4,0,1,0) \\
& (0,4,0,0,0) \circ(0,0,0,4,0)=(0,0,4,4,0,0) \\
& (0,4,0,0,0) \circ(0,0,1,2,0)=(0,0,5,2,0,0) \\
& (0,4,0,0,0) \circ(0,0,3,1,0)=(0,0,7,1,0,0) \\
& (0,4,0,0,0) \circ(0,0,6,0,0)=(0,0,10,0,0,0) \\
& (1,0,0,0,0) \circ(0,0,0,0,1)=(0,1,0,0,1,0) \\
& (1,0,0,0,0) \circ(0,0,0,4,0)=(0,1,0,4,0,0) \\
& (1,0,0,0,0) \circ(0,0,1,2,0)=(0,1,1,2,0,0) \\
& (1,0,0,0,0) \circ(0,0,3,1,0)=(0,1,3,1,0,0) \\
& (1,0,0,0,0) \circ(0,0,6,0,0)=(0,1,6,0,0,0) \\
& (1,0,0,0,0) \circ(0,1,0,1,0)=(0,2,0,1,0,0) \\
& (1,0,0,0,0) \circ(0,1,3,0,0)=(0,2,3,0,0,0) \\
& (1,0,0,0,0) \circ(0,2,1,0,0)=(0,3,1,0,0,0) \\
& (1,0,0,0,0) \circ(0,4,0,0,0)=(0,5,0,0,0,0) \\
& (0,0,0,0,0) \circ(1,0,0,0,0)=(1,0,0,0,0,0)
\end{aligned}
$$

\begin{tabular}{|c|c|c|c|c|c|c|c|}
\hline & & 0 & 1 & 2 & 3 & 4 & 5 \\
\hline 0 & & 1 & 0 & 0 & 0 & 0 & 0 \\
\hline 1 & & 0 & 1 & 3 & 4 & 1 & 0 \\
\hline 2 & & 0 & 0 & 1 & 5 & 4 & 0 \\
\hline 3 & & 0 & 0 & 0 & 1 & 3 & 0 \\
\hline 4 & & 0 & 0 & 0 & 0 & 1 & 0 \\
\hline 5 & & 0 & 0 & 0 & 0 & 0 & 1 \\
\hline & 0 & 1 & 2 & 3 & 4 & 5 & 6 \\
\hline 0 & 1 & 0 & 0 & 0 & 0 & 0 & 0 \\
\hline 1 & 0 & 1 & 4 & 10 & 9 & 1 & 0 \\
\hline 2 & 0 & 0 & 1 & 9 & 24 & 9 & 0 \\
\hline 3 & 0 & 0 & 0 & 1 & 9 & 10 & 0 \\
\hline 4 & 0 & 0 & 0 & 0 & 1 & 4 & 0 \\
\hline 5 & 0 & 0 & 0 & 0 & 0 & 1 & 0 \\
\hline 6 & 0 & 0 & 0 & 0 & 0 & 0 & 1 \\
\hline
\end{tabular}

The notation K1 (n), K2 (n, i, j), K3 (n, i), K4 (n, j) will be used for the number of maximum types having the specified rank, left and right boundaries. Denote by K (n, i, $j$ ) the number of all types of rank $n$ with left boundary $i$ and right boundary $\mathrm{j}$.

For a convenient representation of $K 2(n, i, j)$, of the distribution matrices of maximum types of rank $n$ are used [3]. In them, at the intersection of row $i$ and column $j$, is the element K2 (n, i,j). The sum of the elements of row $i$ is K3 $(n, i)$, the sum of the elements of column $\mathrm{j}$ is $\mathrm{K} 4(\mathrm{n}, \mathrm{j})$ and the sum of all elements of the matrix is K1 (n). In Fig. 1 shows such matrices for ranks 5, 6 and 7 . 


\begin{tabular}{|c|c|c|c|c|c|c|c|c|}
\hline & 0 & 1 & 2 & 3 & 4 & 5 & 6 & 7 \\
\hline 0 & 1 & 0 & 0 & 0 & 0 & 0 & 0 & 0 \\
\hline 1 & 0 & 1 & 5 & 20 & 43 & 25 & 1 & 0 \\
\hline 2 & 0 & 0 & 1 & 14 & 84 & 143 & 25 & 0 \\
\hline 3 & 0 & 0 & 0 & 1 & 19 & 84 & 43 & 0 \\
\hline 4 & 0 & 0 & 0 & 0 & 1 & 14 & 20 & 0 \\
\hline 5 & 0 & 0 & 0 & 0 & 0 & 1 & 5 & 0 \\
\hline 6 & 0 & 0 & 0 & 0 & 0 & 0 & 1 & 0 \\
\hline 7 & 0 & 0 & 0 & 0 & 0 & 0 & 0 & 1 \\
\hline
\end{tabular}

Figure 1. Distribution matrices of types of rank 5, 6 and 7

Next, we show how from distribution matrix of rank $n-1$ can be obtained the distribution matrix of rank $n$

Such matrices we denoted by $\mathrm{M} n$, where $n$ - rank type, the order of such a matrix is n. Figure 1 shows $M_{5}, M_{6}$ and $\mathrm{M}_{7}$.

Let's introduce two operations. The operation "asterisk on the left" denotes the operation of adding a row on the top and the column on the left such: $\left(\begin{array}{cccc}1 & 0 & \ldots & 0 \\ 0 & & & \\ \ldots & & \\ 0 & & \end{array}\right)$, and the operation "asterisk on the right" denotes the operation of adding a row from below and a column on the right such: $\left(\begin{array}{llll} & & 0 \\ & & & \ldots \\ & & & 0 \\ 0 & \ldots & 0 & 1\end{array}\right)$. Let $R_{n}$ denote the upper triangular matrix of order $n+1$ from units, then

$$
\begin{array}{rl}
* R_{n-1} & =\left(\begin{array}{cccccc}
1 & 0 & 0 & 0 & \ldots & 0 \\
0 & 1 & 1 & 1 & \ldots & 1 \\
0 & 0 & 1 & 1 & \ldots & 1 \\
0 & 0 & 0 & 1 & \ldots & 1 \\
\ldots & \ldots & \ldots & \ldots & \ldots & 1 \\
0 & 0 & 0 & 0 & & 1
\end{array}\right) \text {, } \\
R_{n-1} & *=\left(\begin{array}{cccccc}
1 & 1 & 1 & \ldots & 1 & 0 \\
0 & 1 & 1 & \ldots & 1 & 0 \\
0 & 0 & 1 & \ldots & 1 & 0 \\
\ldots & \ldots & \ldots & \ldots & 1 & 0 \\
0 & 0 & 0 & 0 & 1 & 0 \\
0 & 0 & 0 & 0 & 0 & 1
\end{array}\right) .
\end{array}
$$

LEMMA 4. The recurrent matrix equation is valid:

$$
M_{n}=* M_{n-1} \times\left(* R_{n-2} \times M_{n-1}\right) *,
$$

Where the operation $\times$ is the usual matrices multiplication.

Proof. It is easy to verify that the element with indices $(q, j)$ in the inner sum of the matrices product
$* R_{n-2} \times M_{n-1}$ is equal to $\sum_{t=q}^{j} K 2(n-1, t, j)$. Hence, the matrix element $M_{n}$ with indices $(i, j)$, i.e. $K 2(n, i, j)$ is equal to $\sum_{q=i}^{j} K 2(n-1, i-1, q-1) \sum_{t=q}^{j} K 2(n-1, t, j)$. Here, in the external sum, the indices $(i-1, j-1)$ correspond to the row $i$ and the column $j$ of the matrix $M_{n-1}$ shifted to the right by 1 because of the operation of the asterisk on the left. The lemma is proved.

Consequence. Using the upper triangular matrix of units $R_{n-2}$ of dimension $(n-1) \times(n-1)$, we obtain from (1)

$$
M_{n}=* M_{n-1} \times\left(* R_{n-2} \times M_{n-1}\right) *
$$

EXAMPLE 4. We obtain the matrix M6 from the matrix M5.

$$
\begin{aligned}
* R_{4} \times M_{5} & =\left(\begin{array}{cccccc}
1 & 0 & 0 & 0 & 0 & 0 \\
0 & 1 & 1 & 1 & 1 & 1 \\
0 & 0 & 1 & 1 & 1 & 1 \\
0 & 0 & 0 & 1 & 1 & 1 \\
0 & 0 & 0 & 0 & 1 & 1 \\
0 & 0 & 0 & 0 & 0 & 1
\end{array}\right) \times\left(\begin{array}{cccccc}
1 & 0 & 0 & 0 & 0 & 0 \\
0 & 1 & 3 & 4 & 1 & 0 \\
0 & 0 & 1 & 5 & 4 & 0 \\
0 & 0 & 0 & 1 & 3 & 0 \\
0 & 0 & 0 & 0 & 1 & 0 \\
0 & 0 & 0 & 0 & 0 & 1
\end{array}\right)= \\
& =\left(\begin{array}{cccccc}
1 & 0 & 0 & 0 & 0 & 0 \\
0 & 1 & 4 & 10 & 9 & 1 \\
0 & 0 & 1 & 6 & 8 & 1 \\
0 & 0 & 0 & 1 & 4 & 1 \\
0 & 0 & 0 & 0 & 1 & 1 \\
0 & 0 & 0 & 0 & 0 & 1
\end{array}\right)
\end{aligned}
$$$$
* M_{5}=\left(\begin{array}{ccccccc}
1 & 0 & 0 & 0 & 0 & 0 & 0 \\
0 & 1 & 0 & 0 & 0 & 0 & 0 \\
0 & 0 & 1 & 3 & 4 & 1 & 0 \\
0 & 0 & 0 & 1 & 5 & 4 & 0 \\
0 & 0 & 0 & 0 & 1 & 3 & 0 \\
0 & 0 & 0 & 0 & 0 & 1 & 0 \\
0 & 0 & 0 & 0 & 0 & 0 & 1
\end{array}\right),
$$$$
\left(* R_{4} \times M_{5}\right) *\left(\begin{array}{ccccccc}
1 & 0 & 0 & 0 & 0 & 0 & 0 \\
0 & 1 & 4 & 10 & 9 & 1 & 0 \\
0 & 0 & 1 & 6 & 8 & 1 & 0 \\
0 & 0 & 0 & 1 & 4 & 1 & 0 \\
0 & 0 & 0 & 0 & 1 & 1 & 0 \\
0 & 0 & 0 & 0 & 0 & 1 & 0 \\
0 & 0 & 0 & 0 & 0 & 0 & 1
\end{array}\right),
$$ 


$$
\begin{aligned}
M_{6} & =* M_{5} \times\left(* R_{4} \times M_{5}\right) *= \\
& =\left(\begin{array}{ccccccc}
1 & 0 & 0 & 0 & 0 & 0 & 0 \\
0 & 1 & 0 & 0 & 0 & 0 & 0 \\
0 & 0 & 1 & 3 & 4 & 1 & 0 \\
0 & 0 & 0 & 1 & 5 & 4 & 0 \\
0 & 0 & 0 & 0 & 1 & 3 & 0 \\
0 & 0 & 0 & 0 & 0 & 1 & 0 \\
0 & 0 & 0 & 0 & 0 & 0 & 1
\end{array}\right) \times\left(\begin{array}{ccccccc}
1 & 0 & 0 & 0 & 0 & 0 & 0 \\
0 & 1 & 4 & 10 & 9 & 1 & 0 \\
0 & 0 & 1 & 6 & 8 & 1 & 0 \\
0 & 0 & 0 & 1 & 4 & 1 & 0 \\
0 & 0 & 0 & 0 & 1 & 1 & 0 \\
0 & 0 & 0 & 0 & 0 & 1 & 0 \\
0 & 0 & 0 & 0 & 0 & 0 & 1
\end{array}\right)= \\
& =\left(\begin{array}{ccccccc}
1 & 0 & 0 & 0 & 0 & 0 & 0 \\
0 & 1 & 4 & 10 & 9 & 1 & 0 \\
0 & 0 & 1 & 9 & 24 & 9 & 0 \\
0 & 0 & 0 & 1 & 9 & 10 & 0 \\
0 & 0 & 0 & 0 & 1 & 4 & 0 \\
0 & 0 & 0 & 0 & 0 & 1 & 0 \\
0 & 0 & 0 & 0 & 0 & 0 & 1
\end{array}\right)
\end{aligned}
$$

From these operations with matrices, a formula follows for each cell in the distribution table.

$$
K 2(n, i, j)=\sum_{q=i}^{j} K 2(n-1, i-1, q-1) \sum_{t=q}^{j} K 2(n-1, t, j)
$$

The order in equation (1) can be changed.

LEMMA 5. Another matrix equation is valid:

$$
M_{n}=*\left(M_{n-1} \times R_{n-2} *\right) \times M_{n-1} * \text {. }
$$

Proof. From (1), changing a summing order, we have:

$$
\begin{aligned}
& K 2(n, i, j)=\sum_{q=i}^{j} K 2(n-1, i-1, q-1) \sum_{t=q}^{j} K 2(n-1, t, j)= \\
& =\sum_{q=i}^{j} \sum_{t=q}^{j} K 2(n-1, t, j) K 2(n-1, i-1, q-1)= \\
& =\sum_{t=i}^{j} \sum_{q=i}^{t} K 2(n-1, t, j) K 2(n-1, i-1, q-1)= \\
& =\sum_{t=i}^{j} K 2(n-1, t, j) \sum_{q=i}^{t} K 2(n-1, i-1, q-1) .
\end{aligned}
$$

Further, acting in exactly the same way as the proof of Lemma 2, we obtain the assertion of this lemma. The lemma is proved.

Consequence 1. For the number of maximum types $K 2(n, i, j)$ the following expression is valid:

$$
K 2(n, i, j)=\sum_{t=i}^{j} K 2(n-1, t, j) \sum_{q=i}^{t} K 2(n-1, i-1, q-1)
$$

Consequence 2. Using the upper triangular matrix of units $R_{n-2}$ of dimension $(n-1) \times(n-1)$, from (4), we obtain

$$
M_{n}=*\left(M_{n-1} \times R_{n-2} *\right) \times M_{n-1} * .
$$

With increasing rank, the number of types increases significantly.

During the computing of the maximum types, an equality was found between the number of maximum types of MBFs of rank $n$ and all types of rank $n-1$, which significantly reduces the MBFs counting. The study of this dependence topic of the next work.

With types it is more convenient to implement an algorithm for calculating the equivalence classes of nonisomorphic MBFs. The algorithm implemented in [2] calculates some profiles several times, then corrects this action. Our method avoids this. We present the search algorithm for MBF types. Using the previous results, we present below the algorithm in MATLAB, which find are maximum and all types of a given rank. MATLAB is easy to use for processing large vectors and matrices. It has many built-in functions that work well with these types of data and, if desired, further work can be transferred to other programming languages.

The following program finds the number of maximum types of a given rank:

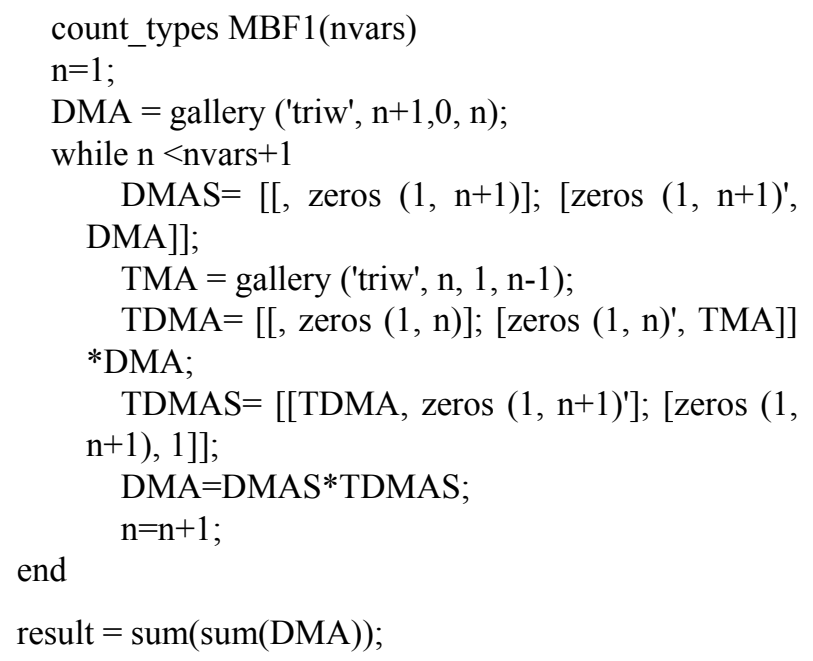

\begin{tabular}{|c|c|c|c|c|c|c|c|c|c|c|}
\hline Rank & 0 & 1 & 2 & 3 & 4 & 5 & 6 & 7 & 8 & 9 \\
\hline Number types & 2 & 3 & 5 & 10 & 26 & 96 & 553 & 5461 & 100709 & 3718354 \\
\hline Number max types & 1 & 2 & 3 & 5 & 10 & 26 & 96 & 553 & 5461 & 100709 \\
\hline \multicolumn{2}{|l|}{ Rank } & \multicolumn{3}{|c|}{10} & \multicolumn{3}{|c|}{11} & \multicolumn{3}{|c|}{12} \\
\hline \multicolumn{2}{|c|}{ Number types } & \multicolumn{3}{|c|}{289725509} & \multicolumn{3}{|c|}{49513793526} & \multicolumn{3}{|c|}{19089032278261} \\
\hline \multicolumn{2}{|c|}{ Number max types } & \multicolumn{3}{|c|}{3718354} & \multicolumn{3}{|c|}{289725509} & \multicolumn{3}{|c|}{49513793526} \\
\hline
\end{tabular}

In the above program, the matrix relations obtained in this work are used.

Table 1. Number of types according to MBF ranks. 
The following program finds all the maximum types of a certain rank:

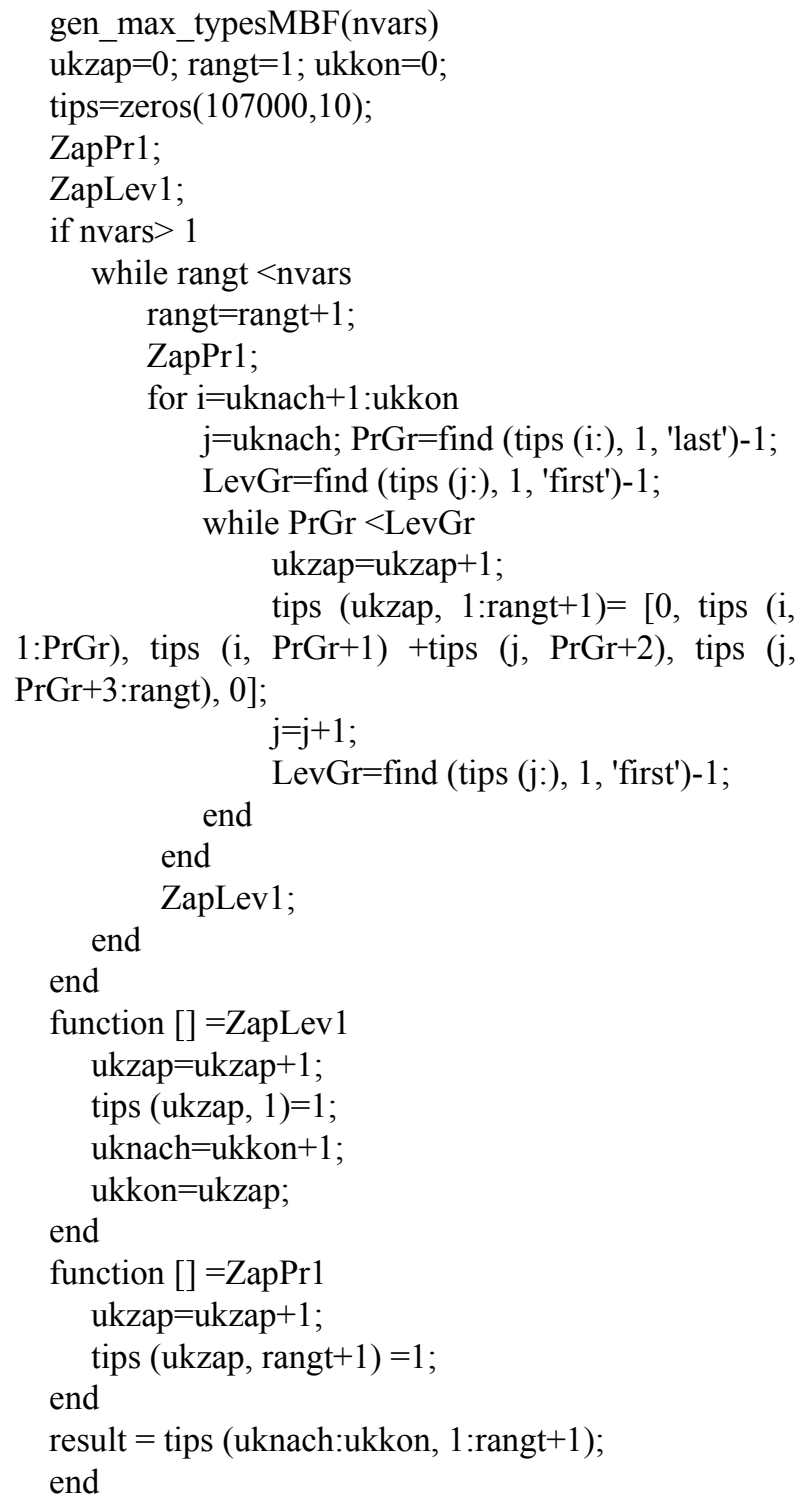

In the above program, the results of Lemmas 1 and 3 are used. It follows from Lemma 1 that for each two types a type of higher rank is obtained, and from Lemma 3 it follows that all these types are distinct.

\section{Conclusions}

In this paper, the notion of type MBF is introduced, the concept of the maximum type, the shift-sum of types, the distribution table of maximum types of MBFs, is introduced for the first time. The paper presents convenient algorithms for search the number of maximum types and the maximum types themselves, and all these types are deduce in lexicographical order.

\section{REFERENCES}

[1] K. Engel, Sperner theory, Cambridge University Press, 1997.

[2] T.Stephen, T.Yusun, Counting inequivalent Monotone Boolean Functions. arXiv: 1209.4623v1 [cs.DS] 20 Sep 2012.

[3] Ткаченко В.Г. Перечисление типов монотонных булевых функций при синтезе цифровых схем. Наукові праці ONAZ ім. O. S. Popova. - 2008. - No. 2. - Page 54 69.

[4] Ткаченко В.Г. Классификация монотонных булевых функций при синтезе цифровых схем. Наукові праці ОНАЗ ім. О.С. Попова, 2008, № 1, 35-43.

[5] Tkachenco V.G., Sinyavsky O.V. Construction of Cryptosystem on the Basis of Triangular Codes, Computer Science and Information Technology, 2 (2014), 300 - 307. DOI: $10.13189 /$ csit.2014.020703.

[6] Tkachenco V.G., Sinyavsky O.V. Recursive Construction of n-gonal Codes on the Basis of Block Design, Computer Science and Information Technology, 2 (2014), 331 - 338. DOI: $10.13189 /$ csit.2014.020803.

[7] Tkachenco V.G., Sinyavsky O.V. Blocks of Monotone Boolean Functions, Computer Science and Information Technology Vol. 4(2), pp. 72 - $78 . \quad$ DOI: $10.13189 /$ csit.2016.040203.

[8] Tkachenco V.G., Sinyavsky O.V. Algebraic Objects of MBFs and Recursive Computation of the Dedekind Number, Computer Science and Information Technology Vol. 5(4), pp. 140 - 147 DOI: 10.13189/csit.2017.050404

[9] Dedekind R. Uber Zerlegungen von Zahlen durch ihre gr" "ossten gemainsamen Teilor. Festschrift Hoch. Braunschweig u. ges. Werke. II. — 1897. — S. 103-148. 